\title{
Investigation of Escherichia coli Selenocysteine Synthase (SelA) Complex Formation Using Cryo-Electron Microscopy (Cryo-EM)
}

Vitor Hugo Balasco Serrão ${ }^{1}$, Lívia Regina Manzine ${ }^{1}$, Ivan Rosa e Silva ${ }^{1}$, Alexandre Cassago ${ }^{2}$, Jefferson Bettini' ${ }^{2}$, Rodrigo Villares Portugal ${ }^{2}$, Marin van Heel $^{3}$ and Otavio Henrique Thiemann ${ }^{1}$

1. São Carlos Physics Institute - University of São Paulo

2. Brazilian Nanotechnology National Laboratory - LNNano - CNPEM

3. Netherlands Centre for Electron Nanoscopy - NeCEN - Leiden Universiteit

Incorporation of selenocysteine (Sec - U) into proteins is directed by a in-frame UGA codon in all domains of life. In Bacteria, Sec biosynthesis and incorporation involves the interaction of Selenocysteine Synthase (SelA), tRNA (SelC or tRNA ${ }^{\mathrm{Sec}}$ ), Selenophosphate Synthetase (SPS), a specific elongation factor known as SelB and the specific mRNA structure SElenocysteine Insertion Sequence (SECIS), forming a complex molecular machinery. SelA is a homodecamer complex responsible for Ser-Sec conversion from selenophosphate delivered by SPS and seryl-tRNA ${ }^{\text {sec }}$, which differs from seryl-tRNA ${ }^{\text {ser }}$ by its long variable arm and the UGA-codon. The specific mRNA sequence known as SElenoCysteine Insertion Sequence forms a hairpin-like secondary structure and is recognized by SelB for Sec incorporation in the nascent peptide [1-3]. Since selenium compounds are highly toxic in cellular environment, selenium association with proteins complexes throughout its metabolism is suggested to be essential for cell survival. However, macromolecular interactions between the different proteins have not yet been characterized.

The objective of this study is to investigate the SelA-tRNA ${ }^{\text {sec }}$ binary complex assembling and the ternary complex formed between SelA-tRNA ${ }^{\text {sec }}$ binary complex and SPS.

The SelA homodecamer and SPS were obtained by overexpression in tRNA $^{\text {sec }}$ deficient $E$. coli WL81460 (DE3) and E. coli BL21 (DE3), respectively. The tRNA ${ }^{\text {sec }}$ was produced by T7 RNA polymerase in vitro transcription. SelA homodecamer and SelA-tRNA ${ }^{\text {sec }}$ binary complex were submitted to transmission electron microscopy analysis with $2 \%$ uranyl acetate negative stain in a JEOL 2100 $\mathrm{LaB}_{6}$ TEM at the Brazilian Nanotechnology National Laboratory (LNNano - CNPEM). Cryo-TEM was also used for the preliminary structural analysis of the SelA homodecamer, the SelA-tRNA ${ }^{\text {sec }}$ binary complex and the SelA-tRNA ${ }^{\mathrm{sec}}$-SelD ternary complex. Sample preparation was made by rapid freezing in liquid ethane cooled by liquid nitrogen and vitrified by a Vitrobot FEI - Mark IV system. Particles were visualized in the same microscope.

We observed supramolecular stacking of SelA homodecamers only in the absence of tRNA ${ }^{\text {sec }}$, consistent with previous fluorescence anisotropy spectroscopy experiments [4]. The use of Principal Component Analysis (PCA) and a Multivariate Statistical Analysis (MSA) on 7582 negative stained SelA-tRNA ${ }^{\mathrm{sec}}$ particles showed, by eigenimages analysis, a fivefold symmetry resulting in a D5 pointgroup symmetry structure. This result agrees with $1 \mathrm{SelA}: 1 \mathrm{tRNA}^{\mathrm{sec}}$ stoichiometric ratio for the binary complex. Additionally, Cryo-TEM showed that SelA-tRNA ${ }^{\text {sec }}$ binary complex have dimensions compatible with dimeric SPS interaction, considering the SPS dimensions recently observed by X-ray crystallography [5]. Our results confirm the overall structure of the binary complex, recently published by Itoh et al. [6], adding novel information on the complex formation and in selenium delivery in the bacteria. 
Together, our results give new insights into the selenium delivery system in the selenocysteine incorporation pathway and we propose the mechanism by which Sec biosynthesis takes place in the tRNA $^{\text {sec }}$.

[1] STADTMAN, T.C., PloS Biology, 3 (2005) e421.

[2] BOCK, A., Selenium, (2001) 7-422.

[3] FORCHHAMMER, K. et al, Nature, (1989) 453.

[4] MANZINE, L.R., et al, FEBS Letters, 587 (2013) 906.

[5] NOINAJ, N., et al, Journal of Bacteriology, 194 (2012) 499-508.

[6] ITOH, Y., et al, Science, 340 (2013) 75.

[7] The authors acknowledge funding from The National Research Council (CNPq), the "Science without border" program and the São Paulo State Funding Agency (FAPESP).
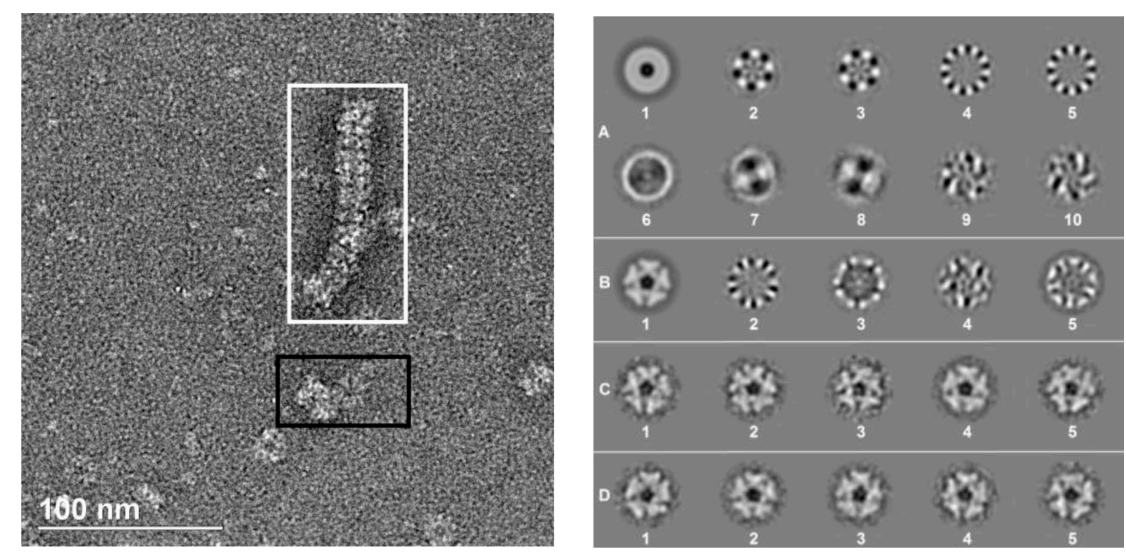

Figure 1 - NS-TEM of SelA and SelA-tRNA ${ }^{\text {sec }}$ binary complex. Left - EM image of the negative stained SelA sample. The white box shows a supramolecular stack-structure and the black box a side-by-side assembly of SelA homodecamers. Right - Electron microscopical analysis of negatively stained complexes using PCA. (A) First ten eigenimages obtained. Eigenimages A2 and A3 show the underlying D5 symmetry of this dataset. (B) First 5 eigenimages of PCA of "top" views of the dataset after rotational alignment to the second eigenimage of the dataset - A2. (C) Class averages of the unbound state of SelA. (D) Class averages of the SelA-tRNA ${ }^{\mathrm{sec}}$ binary complex.
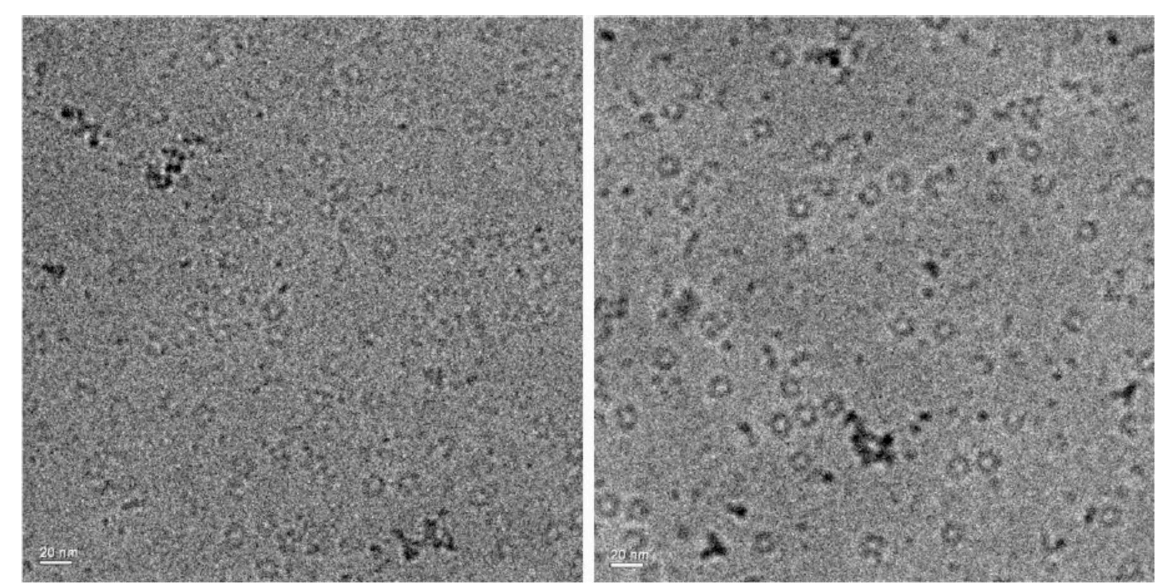

Figure 2 - Cryo-EM of SelA. Homodecameric SelA ring-shapped images observed by Cryo-TEM. 